\title{
Hauser-Feshbach reaction rates with parity-dependent level densities
}

\author{
H.P. Loens ${ }^{* a, b}$, E. Litvinova ${ }^{a}$, K. Langanke ${ }^{a, b}$, G. Martínez-Pinedo $^{a}$, T. Rauscher ${ }^{c}$, \\ F.-K. Thielemann ${ }^{c}$, P. Ring ${ }^{d}$, and V. Tselyaev ${ }^{e}$ \\ ${ }^{a}$ Gesellschaft für Schwerionenforschung mbH, 64291 Darmstadt (Germany) \\ ${ }^{b}$ Technische Universität Darmstadt, Institut für Kernphysik, 64289 Darmstadt (Germany) \\ ${ }^{c}$ Universität Basel, 4056 Basel (Switzerland) \\ ${ }^{d}$ Physik-Department, Technische Universität München, 85748 Garching (Germany) \\ ${ }^{e}$ Nuclear Physics Department, V. A. Flock Institute of Physics, St. Petersburg State University, \\ 198504 St. Petersburg (Russia) \\ Email: H.P.Loensegsi.de
}

\begin{abstract}
This manuscript deals with the influence of parity-dependent nuclear level densities on astrophysical reaction rates calculated in the Hauser-Feshbach framework. The inclusion of a parity non-equipartition within the compound nucleus in these reactions has not been examined before. We show that our approximative treatment - that accounts for such non-uniformly distributed parities in the compound nucleus - can have an influence on reaction rates. For a more detailed examination we refer to the corresponding paper [1].

Moreover, we shortly discuss the influence of microscopic calculated E1-strength functions on reaction rates and show that for specific nuclei, additional low-lying E1 strength can increase the reaction rate.
\end{abstract}

10th Symposium on Nuclei in the Cosmos

July 27 - August 1, 2008

Mackinac Island, Michigan, USA

\footnotetext{
*Speaker.
} 


\section{Introduction}

Stellar nucleosynthesis, e.g. explosive nuclear burning, involves a very large amount of nuclei, which have not been fully explored by experiments yet. Therefore one needs to predict the reaction cross sections and thermonuclear reactions rates by using theoretical models. The most widely used approach is the so-called statistical or Hauser-Feshbach [2] model that describes the reaction going via the formation of a compound nucleus. The main idea of this model is the fact that for high nuclear level densities it is not possible to resolve resonances belonging to certain states. Therefore, one can average over these resonances and obtain the reaction cross section without specific knowledge of the resonances [3, 4, 5, 6. Consequently, the applicability of this model is strongly related to the total level density [6]. In case of explosive nucleosynthesis the relevant reactions include nuclei with masses larger than $A \gtrsim 30$ and accordingly these nuclei have a high density of excited states compared to nuclei with lesser nucleons. The Hauser-Feshbach model is therefore applicable for a large set of reactions.

\section{Nuclear Level Densities}

There are several different approaches to calculate the nuclear level density, all varying in their complexity, applicability and quality. There are analytical models based upon the statistical Fermi gas picture of the nucleus combined with phenomenological parameters (see [6, 7] and references therein). Alternatively, there are microscopic models based on shell-model Monte Carlo (匹8] and references therein) or Hartree-Fock-BCS [9]. The advantage of these microscopic approaches is the possibility to calculate the level density not only depending on excitation energy $E$ but also depending on spin $J$ and parity $\pi$. Concerning the parity, it has been assumed that both parities are equally given in any nucleus at any energy. This approximation is certainly true for most nuclei at excitation energies above several $\mathrm{MeV}$. Therefore, this is a good assumption for capture reactions involving a compound nucleus with a large separation energy of the captured particle. However, recent theoretical calculations and experimental studies showed that this assumption of equipartitioned parities is not always true even for certain nuclei up to rather high excitation energies (see [1] for more details and a list of references). Since the influence of this non-equipartition within the target and the residual nucleus has been examined in [10], we go one step further: we additionally introduce a parity non-equipartition also within the compound nucleus (see [1]).

\subsection{Parity-dependence in the Statistical Model}

The Hauser-Feshbach model makes use of the so-called transmission coefficients $T_{\text {in }}(E, J, \pi ; a)$ and $T_{\text {out }}(E, J, \pi ; b)$ 1111] describing the entrance channel and the exit channel. The quantum numbers $(E, J, \pi)$ are related to the compound nucleus. The variables $a$ and $b$ reflect the additional quantum numbers for the target and the residual nucleus. These transmission coefficients are subject to quantum mechanical selection rules and should be obtained from a microscopic approach. However, no such models exist (for more details see [1]). The only globally applicable model is the optical model which only gives an energy dependence and a trivial dependence on angular momentum due to the centrifugal barrier. 
In order to account for the deficiency of a lacking parity dependence in the optical model, we intend to introduce a parity dependence within the compound nucleus and therefore we modify the transmission coefficients in the following way:

$$
T(E, J, \pi) \longrightarrow \beta(E, \pi ; J) T(E, J, \pi)
$$

with the $\beta$ defined by

$$
\beta(E, \pi ; J)=\frac{2 \cdot \rho_{\mathrm{cmp} .}(E, J, \pi)}{\sum_{\pi} \rho_{\mathrm{cmp} .}(E, J, \pi)} .
$$

Here we used the observation, that for the average transmission coefficients, there is the linear relation $T \propto\langle\Gamma\rangle / D$, involving the level spacing $D=1 / \rho$ and the average level width $\langle\Gamma\rangle$ in the considered reaction channel. The $\rho(E, J, \pi)$ have to come from a method that resolves the parity dependence [8, 10, 12, 13].

\section{3. $\gamma$-strength Functions}

Besides the nuclear level density, one also needs other important nuclear input such as masses, optical models and strength functions. In case of $\gamma$-decays one needs the strength-functions for the considered transitions types. In our case, the only considered transitions are E1 and M1 transitions. The E1 transitions are more likely than the M1 transitions since they are of electric type and have the same multipolarity $L=1^{1}$. However, E1 transitions and M1 transitions differ concerning the conservation of parity: E1 transitions change the parity while M1 transitions conserve it.

In general one calculates the according transmission coefficients in the following way ( $\mathrm{X} \hat{=}$ type of transition; $\mathrm{L} \hat{=}$ multipolarity):

$$
T_{\mathrm{XL}}\left(E_{\gamma}\right)=2 \pi E_{\gamma}^{2 L+1} f_{\mathrm{XL}}\left(E_{\gamma}\right)
$$

with $f_{\mathrm{XL}}$ being the according strength-function. The latter can be calculated in different ways: The E1 strength function $f_{\mathrm{E} 1}$ is usually calculated based upon the Lorentzian description of the giant dipole resonance. However, this approach is known to be less good at low energies [14] 7] and it cannot reproduce possible additional low-lying strength. An alternative is therefore to calculate the strength function in a microscopical model which can resolve these structures. These calculations are based on the Quasiparticle Random Phase Approximation on top of Hartree-Fock-Bogoliubov [15] 16] or relativistic mean-field models (RQRPA) [17]. Litvinova et al. recently extended the RQRPA (see [18]) to include quasiparticle-phonon coupling. This approach reproduces experimental strength distributions very well, including observed additional low-lying strength [18]. The M1 transitions are usually calculated in the single-particle or Weisskopf approach, $f_{\mathrm{M} 1}=$ constant. An alternative approach uses a phenomenological Lorentzian distribution of the M1 strength [7] with the parameters $E_{\mathrm{M} 1}=41 A^{-1 / 3}$ and $\Gamma=4 \mathrm{MeV}$. We will restrict ourselves to the use of the single-particle model for M1 transitions in this paper.

\footnotetext{
${ }^{1}$ Magnetic transitions are smaller than electric transitions of the same multipolarity. At the same time transitions of the same type (magnetic or electric) are less likely with increasing multipolarity.
} 


\section{Results}

In the following we will restrict ourselves to neutron capture reactions, and in particular to $(n, \gamma)$-reactions. The relevant energy scale for these reactions is the neutron separation energy $S_{n}$.

\subsection{Parity-dependence in the Compound Nucleus}

In Fig. 11 we can see the influence of the parity treatment on the reaction rate for $(n, \gamma)$ reactions. We used two different sets of parity-distribution: the one from Hilaire et al. [13] and the one from Mocelj et al. [10]. Both distributions give nearly the same behavior.
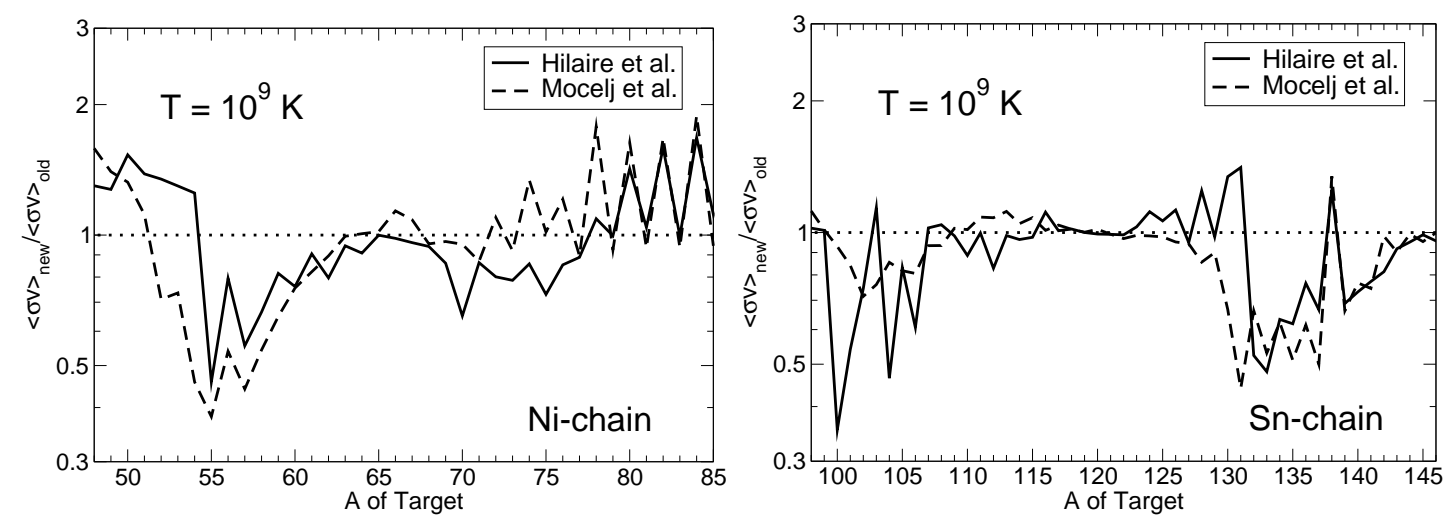

Figure 1: Ratio of the reaction rate using a full parity dependence to the reaction rate using equipartitioned parities in several nickel isotopes. The reaction rates are calculated for $\mathrm{T}=10^{9} \mathrm{~K}$.

We can see an odd-even effect resulting from pairing, since an even A compound nucleus has a larger neutron separation energy (and is therefore higher excited) than its odd A neighbor nuclei. If the excitation energy is higher, $\beta$ is closer to unity than for lower energies and the ratio in Fig. 1 also approaches unity. At the neutron rich side of the nickel isotopes, we see an increment: this results from the constant single-particle M1 strength which becomes larger than the E1 strength (which has a Lorentzian shape) for low $\gamma$-energies and these nuclei are dominated by M1 transitions since positive parity dominates up to rather high energies (due to the filling of the $g_{9 / 2}$ and $g_{7 / 2}$ orbits). Therefore the parity conserving M1 transitions - that are larger are larger at these low energies because they are calculated using the single-particle approach - are favored. This implies that an improved description of the M1 transitions is needed in the vicinity of the introduced parity treatment.

Concerning the tin isotopes we do not see large differences between both parity distributions again. However, the shell-structure in tin is more complicated due to the $h_{11 / 2}$ intruder orbit. For a more extensive discussion about the results refer to Ref. [1].

\subsection{Microscopic $\gamma$-strength Functions}

As we can see in Fig. 2, the influence of low-lying E1 strength on the reaction rate and the cross section depends on the location of this additional strength relative to the neutron threshold. If the additional strength is located just around the threshold, it can strongly increase the cross section and reaction rate. We will discuss this in an upcoming manuscript in more detail. 

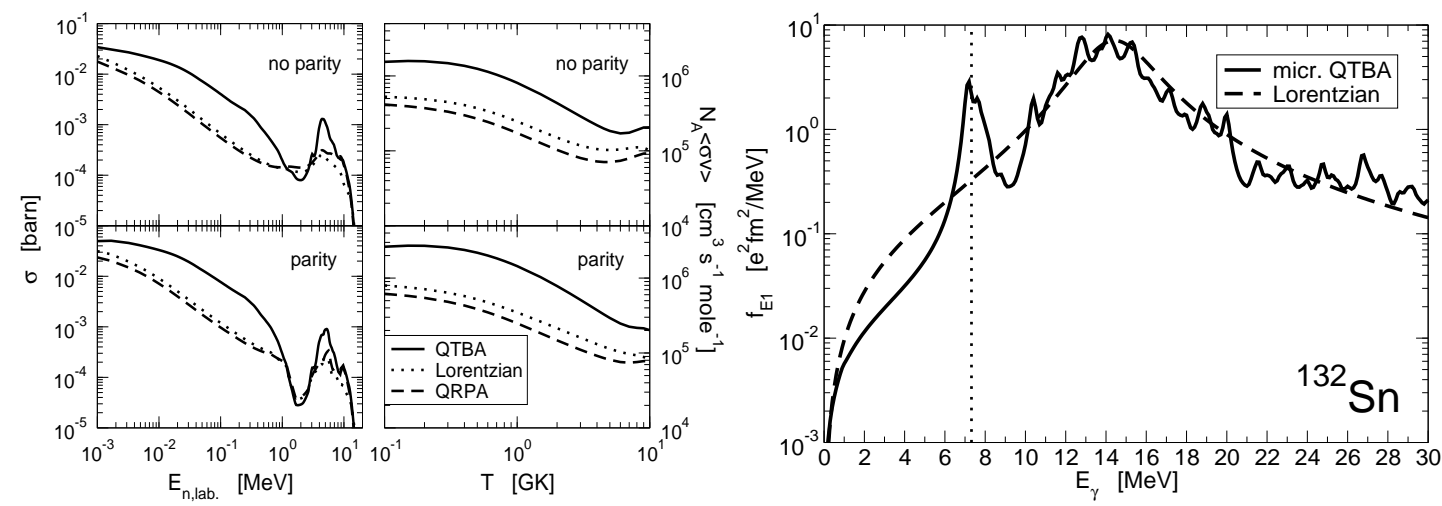

Figure 2: Laboratory cross-sections and stellar reaction rates (left panel) for the reaction ${ }^{131} \operatorname{Sn}(n, \gamma){ }^{132} \mathrm{Sn}$ using three different prescriptions for the $f_{\mathrm{E} 1}$ (see Eq. 3.1p. For the calculation labelled "QTBA" we used the microscopic strength function obtained in the RQTBA [18], "Lorentz" is a simple Lorentzian prescription normalized to the total strength of the "QTBA" function, and "QRPA" the microscopic strength function of [15]. "parity" and "no parity" are calculations either using our parity treatment introduced above or without using any parity dependence at all. The right panel shows the comparison between the "QTBA" and the "Lorentz" strength function. The vertical line marks the neutron separation energy in ${ }^{132} \mathrm{Sn}$.

\section{References}

[1] H. P. Loens, K. Langanke, G. Martínez-Pinedo, T. Rauscher, F.-K. Thielemann, Phys. Lett. B 666 (2008) (4) 395.

[2] W. Hauser, H. Feshbach, Phys. Rev. 87 (1952) (2) 366.

[3] J. A. Holmes, S. E. Woosley, W. A. Fowler, B. A. Zimmerman, At. Data Nucl. Data Tables 18 (1976) 305.

[4] J. J. Cowan, F.-K. Thielemann, J. W. Truran, Phys. Rep. 208 (1991) (4/5).

[5] E. Gadioli, P. E. Hodgson, Pre-Equilibrium Nuclear Reactions (Clarendon Press Oxford, 1992).

[6] T. Rauscher, F.-K. Thielemann, K.-L. Kratz, Phys. Rev. C 56 (1997) (3) 1613.

[7] T. Belgya, et al., Handbook for calculations of nuclear reaction data, RIPL-2, IAEA-TECDOC-1506 (IAEA, Vienna, 2006), available online at http://www-nds.iaea.org/RIPL-2/.

[8] C. Özen, K. Langanke, G. Martínez-Pinedo, D. J. Dean, Phys. Rev. C 75 (2007) (6) 064307.

[9] P. Demetriou, S. Goriely, Nucl. Phys. A 695 (2001) 95.

[10] D. Mocelj, et al., Phys. Rev. C 75 (2007) (4) 045805.

[11] T. Rauscher, F.-K. Thielemann, At. Data Nucl. Data Tables 75 (2000) 1.

[12] H. Nakada, Y. Alhassid, Phys. Rev. Lett. 79 (1997) (16) 2939.

[13] S. Hilaire, S. Goriely, Nucl. Phys. A 779 (2006) 63.

[14] J. Kopecky, M. Uhl, Phys. Rev. C 41 (1990) (5) 1941.

[15] S. Goriely, E. Khan, Nucl. Phys. A 706 (2002) 217.

[16] S. Goriely, E. Khan, M. Samyn, Nucl. Phys. A 739 (2004) 331.

[17] N. Paar, P. Ring, T. Nikšić, D. Vretenar, Phys. Rev. C 67 (2003) 034312.

[18] E. Litvinova, P. Ring, V. Tselyaev, Phys. Rev. C 78 (2008) 014312. 